\title{
Complex Wave Solutions to Mathematical Biology Models I: Newell-Whitehead-Segel and Zeldovich Equations
}

\author{
Alper Korkmaz ${ }^{a, *}$ \\ ${ }^{a}$ Çankırı Karatekin University, Department of Mathematics, 18200, Çankırı, Turkey.
}

\begin{abstract}
Complex and real valued exact solutions to some reaction-diffusion equations are suggested by using homogeneous balance and Sine-Gordon equation expansion method. The predicted solution of finite series of some hyperbolic functions are determined by using some relations between the hyperbolic functions and trigonometric functions based on Sine-Gordon equation and traveling wave transform. The Newel-Whitehead-Segel and Zeldovich equations are solved explicitly. Some real valued solutions are depicted for some particular choice of parameters.
\end{abstract}

Keywords: Reaction-Diffusion Equations; Newell-Whitehead-Segel Equation; Zeldovich Equation; Exact Solution, Traveling Wave Solution.

MSC2010: 35C07;35R11;35Q53.

PACS: 02.30.Jr; 02.70.Wz; 04.20.Jb

\section{Introduction}

The Newel-Whitehead-Segel equation (NWSE), one of the significant concepts of pattern formation theory, (sometimes it is called only Newel-Whitehead Equation, amplitude equation [1] or a particular form of the Chafee-Infante equation with constant coefficients in the literature) in a parametrically generalized onedimensional form can be defined as

$$
u_{t}+p u_{z z}+q u+r u^{3}=0
$$

*alperkorkmaz7@gmail.com 
where $p$ is diffusion coefficient, $q$ and $r$ are parameters related to reaction terms, $u=u(z, t)$ and the subscripts denote partial derivatives of dependent function. The choice $r=-q$ gives the equation in its firstly defined form [2,3]. Here, we suppose $r$ to be a free parameter. The model (1) was proposed to explain the relation between a continuous finite bandwidth of modes and post-critical Rayleigh-Benard convection with the aid of space-time tardily changing amplitudes [2]. The same model was also suggested to describe vertical boundary effects of thermal convection in a shallow water heated from below by Segel [3]. Some point defect and grain boundary problems were described by the use of framework of this equation [4-6]. The stability conditions and grain boundaries of the NWSE were deeply examined by Malomed [7]. A variation of the NWSE under random rotations was constructed by renormalization group technique [8]. Nonlinear analysis of stripped patterns in two dimension is another way to reach the NWSE [9]. The structure of its attractor of this parabolic PDE is one of the almost completely understood model [10].

A comparative study on the NWSE puts forward the semi analytical-numerical in series forms of the solutions [11]. Various solutions some problems covering IVPs were determined by He's method variational iteration approach [12].

The second equation considered in this study is the Zeldovich equation (ZE) of the form

$$
u_{t}+p u_{z z}+q u^{2}+r u^{3}=0
$$

where $u=u(z, t), p$ is coefficient of transport and $q$ and $r$ are some particular parameters having critical roles on population evolution [13]. The ZE (2) describes evolution of a grove population where $q$ and $r$ are resource distributions that controls vital phenomena like birth and death of the population [13]. One should necessarily note that the biological model has significant similarities in the combustion model in chemistry $[1,13]$. Localized solutions of the ZE (2) are the most important difference between the population and combustion models. Without dealing with biological or chemical models, this study aims to construct complex solutions to both equations by using some compatible wave transforms. The finite series of multiplications of powers of some hyperbolic functions are main forms of the solutions. The solutions are determined by using general constant parameters without any limitations if not necessary. Before starting construction of the exact solutions, some mathematical preliminaries on solution technique are represented in the next section. The solutions are explicitly represented in the following sections for both equations. 


\section{Expansion Method based on Sine-Gordon Equa- tion}

Let implement the one dimensional traveling wave transform

$$
\begin{gathered}
u(z, t) \rightarrow U(\eta) \\
\eta=a(z-\nu t)
\end{gathered}
$$

to the one dimensional Sine-Gordon Equation

$$
u_{z z}-u_{t t}=m^{2} \sin u
$$

where $u=u(z, t), m$ is constant coefficient. The resultant equation of one independent variable can be written as

$$
\frac{d^{2} U}{d \eta^{2}}=\frac{m^{2}}{a^{2}\left(1-\nu^{2}\right)} \sin U
$$

where $\nu$ defines the propagation velocity of the traveling wave [14]. Some calculus leads

$$
\left(\frac{d(U / 2)}{d \eta}\right)^{2}=\frac{m^{2}}{a^{2}\left(1-\nu^{2}\right)} \sin ^{2} U / 2+C
$$

where $C=0$ is integration constant. The change of variable $w(\eta)=U(\eta) / 2$ and assuming $m^{2} /\left(a^{2}\left(1-\nu^{2}\right)\right)=1$ converts (6) to

$$
\frac{d(w)}{d \eta}=\sin w
$$

Solution of this differential equation yields the relations

$$
\sin w(\eta)=\left.\frac{2 c e^{\eta}}{c^{2} e^{2 \eta}+1}\right|_{c=1}=\operatorname{sech} \eta
$$

and

$$
\cos w(\eta)=\left.\frac{c^{2} e^{2 \eta}-1}{c^{2} e^{2 \eta}+1}\right|_{c=1}=-\tanh \eta
$$

between $w$ and $\eta$.

The governing PDE (1) or (2) is reduced to an ODE of the form

$$
\tilde{\Omega}\left(U, U^{\prime}, U^{\prime \prime}, \ldots\right)=0
$$

where ' denotes derivative $d U / d \eta$ by the wave transform (3). 
Now, assume that (10) has some solutions in the form

$$
U(\eta)=A_{0}+\sum_{i=1}^{s} \tanh ^{i-1}(\eta)\left(B_{i} \operatorname{sech} \eta+A_{i} \tanh \eta\right)
$$

where $s$ is index limit and $A_{i}$ and $B_{i}$ are the coefficients to be determined with the property $A_{s} \neq 0$ or $B_{s} \neq 0$.

The from of this predicted solution can be written in trigonometric functions of $w$ as

$$
U(w)=A_{0}+\sum_{i=1}^{s}-\cos ^{i-1}(w)\left(B_{i} \sin w-A_{i} \cos w\right)
$$

owing to the relations (8) - (9) by the Sine-Gordon equation. The first step is to find $s$ by the help of balancing the highest ordered derivative and nonlinear terms. Once $s$ is determined, the predicted solution is substituted into the ODE (10) and equating the coefficients of trigonometric functions and remaining coefficients to zero gives a set of algebraic equations. The solution of this system for target coefficients $A_{0}, A_{1}, B_{1}, \ldots$ and any or both of nonzero transform parameters $a$ and $\nu$ is the key to construct the solutions.

\section{Solutions to the NWSE}

The traveling wave transform (3) reduces the NWSE (1) to

$$
-a \nu U^{\prime}+p a^{2} U^{\prime \prime}+q U+r U^{3}=0
$$

where ' denotes $d U / d \eta$. It is easy to see that the balance between $U^{\prime \prime}$ and $U^{3}$ gives $s=1$. Thus, the predicted solution to (13) takes the form

$$
U(\eta)=A_{0}+A_{1} \tanh \eta+B_{1} \operatorname{sech} \eta
$$

or

$$
U(w)=A_{0}-A_{1} \cos w+B_{1} \sin w
$$

Substitution of this solution into (13) and equating the powers of multiplication of $\sin$ and $\cos$ functions to zero lead the system of algebraic equations

$$
\begin{aligned}
r A_{0}{ }^{3}+3 r A_{0} B_{1}{ }^{2}-a \nu A_{1}+q A_{0} & =0 \\
-a^{2} p B_{1}+3 r A_{0}{ }^{2} B_{1}+r B_{1}{ }^{3}+q B_{1} & =0 \\
2 a^{2} p B_{1}+3 r A_{1}{ }^{2} B_{1}-r B_{1}{ }^{3} & =0 \\
-6 r A_{0} A_{1} B_{1}-a \nu B_{1} & =0 \\
-2 a^{2} p A_{1}-r A_{1}{ }^{3}+3 r A_{1} B_{1}{ }^{2} & =0 \\
3 r A_{0} A_{1}{ }^{2}-3 r A_{0} B_{1}{ }^{2}+a \nu A_{1} & =0 \\
2 a^{2} p A_{1}-3 r A_{0}{ }^{2} A_{1}-3 r A_{1} B_{1}{ }^{2}-q A_{1} & =0
\end{aligned}
$$


Solution of this system for $A_{0}, A_{1}, B_{1}, a \neq 0$ and $\nu \neq 0$ satisfying the condition that at least one of $A_{1}$ and $B_{1}$ is non zero gives the solution sets

$$
\begin{aligned}
& a=\sqrt{\frac{q}{2 p}}, \quad \nu=\frac{3 \sqrt{p q}}{\sqrt{2}}, A_{0}=\sqrt{-\frac{q}{4 r}}, \quad A_{1}=\sqrt{-\frac{q}{4 r}}, B_{1}=\sqrt{\frac{q}{4 r}} \\
& a=\sqrt{\frac{q}{2 p}}, \quad \nu=-\frac{3 \sqrt{p q}}{\sqrt{2}}, A_{0}=-\sqrt{-\frac{q}{4 r}}, \quad A_{1}=\sqrt{-\frac{q}{4 r}}, B_{1}=\sqrt{\frac{q}{4 r}} \\
& a=\sqrt{\frac{q}{2 p}}, \quad \nu=-\frac{3 \sqrt{p q}}{\sqrt{2}}, A_{0}=\sqrt{-\frac{q}{4 r}}, \quad A_{1}=-\sqrt{-\frac{q}{4 r}}, B_{1}=\sqrt{\frac{q}{4 r}} \\
& a=\sqrt{\frac{q}{2 p}}, \quad \nu=\frac{3 \sqrt{p q}}{\sqrt{2}}, A_{0}=-\sqrt{-\frac{q}{4 r}}, \quad A_{1}=-\sqrt{-\frac{q}{4 r}}, B_{1}=\sqrt{\frac{q}{4 r}} \\
& a=-\sqrt{\frac{q}{2 p}}, \quad \nu=-\frac{3 \sqrt{p q}}{\sqrt{2}}, A_{0}=\sqrt{-\frac{q}{4 r}}, \quad A_{1}=\sqrt{-\frac{q}{4 r}}, B_{1}=\sqrt{\frac{q}{4 r}} \\
& a=-\sqrt{\frac{q}{2 p}}, \quad \nu=\frac{3 \sqrt{p q}}{\sqrt{2}}, A_{0}=-\sqrt{-\frac{q}{4 r}}, \quad A_{1}=\sqrt{-\frac{q}{4 r}}, B_{1}=\sqrt{\frac{q}{4 r}} \\
& a=-\sqrt{\frac{q}{2 p}}, \quad \nu=\frac{3 \sqrt{p q}}{\sqrt{2}}, A_{0}=\sqrt{-\frac{q}{4 r}}, \quad A_{1}=-\sqrt{-\frac{q}{4 r}}, B_{1}=\sqrt{\frac{q}{4 r}} \\
& a=-\sqrt{\frac{q}{2 p}}, \quad \nu=-\frac{3 \sqrt{p q}}{\sqrt{2}}, A_{0}=-\sqrt{-\frac{q}{4 r}}, \quad A_{1}=-\sqrt{-\frac{q}{4 r}}, B_{1}=\sqrt{\frac{q}{4 r}} \\
& a=\sqrt{\frac{q}{2 p}}, \quad \nu=\frac{3 \sqrt{p q}}{\sqrt{2}}, A_{0}=\sqrt{-\frac{q}{4 r}}, \quad A_{1}=\sqrt{-\frac{q}{4 r}}, B_{1}=-\sqrt{\frac{q}{4 r}} \\
& a=\sqrt{\frac{q}{2 p}}, \quad \nu=-\frac{3 \sqrt{p q}}{\sqrt{2}}, A_{0}=-\sqrt{-\frac{q}{4 r}}, \quad A_{1}=\sqrt{-\frac{q}{4 r}}, B_{1}=-\sqrt{\frac{q}{4 r}} \\
& a=\sqrt{\frac{q}{2 p}}, \quad \nu=-\frac{3 \sqrt{p q}}{\sqrt{2}}, A_{0}=\sqrt{-\frac{q}{4 r}}, \quad A_{1}=-\sqrt{-\frac{q}{4 r}}, B_{1}=-\sqrt{\frac{q}{4 r}} \\
& a=\sqrt{\frac{q}{2 p}}, \quad \nu=\frac{3 \sqrt{p q}}{\sqrt{2}}, A_{0}=-\sqrt{-\frac{q}{4 r}}, \quad A_{1}=-\sqrt{-\frac{q}{4 r}}, B_{1}=-\sqrt{\frac{q}{4 r}} \\
& a=-\sqrt{\frac{q}{2 p}}, \quad \nu=-\frac{3 \sqrt{p q}}{\sqrt{2}}, A_{0}=\sqrt{-\frac{q}{4 r}}, \quad A_{1}=\sqrt{-\frac{q}{4 r}}, B_{1}=-\sqrt{\frac{q}{4 r}} \\
& a=-\sqrt{\frac{q}{2 p}}, \quad \nu=\frac{3 \sqrt{p q}}{\sqrt{2}}, A_{0}=-\sqrt{-\frac{q}{4 r}}, \quad A_{1}=\sqrt{-\frac{q}{4 r}}, B_{1}=-\sqrt{\frac{q}{4 r}} \\
& a=-\sqrt{\frac{q}{2 p}}, \quad \nu=\frac{3 \sqrt{p q}}{\sqrt{2}}, A_{0}=\sqrt{-\frac{q}{4 r}}, \quad A_{1}=-\sqrt{-\frac{q}{4 r}}, B_{1}=-\sqrt{\frac{q}{4 r}} \\
& a=-\sqrt{\frac{q}{2 p}}, \quad \nu=-\frac{3 \sqrt{p q}}{\sqrt{2}}, A_{0}=-\sqrt{-\frac{q}{4 r}}, \quad A_{1}=-\sqrt{-\frac{q}{4 r}}, B_{1}=-\sqrt{\frac{q}{4 r}} \\
& a=\sqrt{\frac{q}{8 p}}, \quad \nu=\frac{3 \sqrt{p q}}{\sqrt{2}}, A_{0}=\sqrt{-\frac{q}{4 r}}, \quad A_{1}=\sqrt{-\frac{q}{4 r}}, B_{1}=0 \\
& a=\sqrt{\frac{q}{8 p}}, \quad \nu=-\frac{3 \sqrt{p q}}{\sqrt{2}}, A_{0}=-\sqrt{-\frac{q}{4 r}}, \quad A_{1}=\sqrt{-\frac{q}{4 r}}, B_{1}=0 \\
& a=\sqrt{\frac{q}{8 p}}, \quad \nu=-\frac{3 \sqrt{p q}}{\sqrt{2}}, A_{0}=\sqrt{-\frac{q}{4 r}}, \quad A_{1}=-\sqrt{-\frac{q}{4 r}}, B_{1}=0 \\
& a=\sqrt{\frac{q}{8 p}}, \quad \nu=\frac{3 \sqrt{p q}}{\sqrt{2}}, A_{0}=-\sqrt{-\frac{q}{4 r}}, \quad A_{1}=-\sqrt{-\frac{q}{4 r}}, B_{1}=0 \\
& a=-\sqrt{\frac{q}{8 p}}, \quad \nu=\frac{3 \sqrt{p q}}{\sqrt{2}}, A_{0}=\sqrt{-\frac{q}{4 r}}, \quad A_{1}=\sqrt{-\frac{q}{4 r}}, B_{1}=0 \\
& a=-\sqrt{\frac{q}{8 p}}, \quad \nu=-\frac{3 \sqrt{p q}}{\sqrt{2}}, A_{0}=-\sqrt{-\frac{q}{4 r}}, \quad A_{1}=\sqrt{-\frac{q}{4 r}}, B_{1}=0 \\
& a=-\sqrt{\frac{q}{8 p}}, \quad \nu=-\frac{3 \sqrt{p q}}{\sqrt{2}}, A_{0}=\sqrt{-\frac{q}{4 r}}, \quad A_{1}=-\sqrt{-\frac{q}{4 r}}, B_{1}=0 \\
& a=-\sqrt{\frac{q}{8 p}}, \quad \nu=\frac{3 \sqrt{p q}}{\sqrt{2}}, A_{0}=-\sqrt{-\frac{q}{4 r}}, \quad A_{1}=-\sqrt{-\frac{q}{4 r}}, B_{1}=0
\end{aligned}
$$


Using these relations, the solutions to (1) are written in the forms

$$
\begin{aligned}
& u_{1}(z, t)=\sqrt{-\frac{q}{4 r}}+\sqrt{-\frac{q}{4 r}} \tanh a(z-\nu t)+\sqrt{\frac{q}{4 r}} \operatorname{sech} a(z-\nu t), \\
& a=\sqrt{\frac{q}{2 p}}, \nu=\frac{3 \sqrt{p q}}{\sqrt{2}} \\
& u_{2}(z, t)=-\sqrt{-\frac{q}{4 r}}+\sqrt{-\frac{q}{4 r}} \tanh a(z-\nu t)+\sqrt{\frac{q}{4 r}} \operatorname{sech} a(z-\nu t), \\
& a=\sqrt{\frac{q}{2 p}}, \nu=-\frac{3 \sqrt{p q}}{\sqrt{2}} \\
& u_{3}(z, t)=\sqrt{-\frac{q}{4 r}}-\sqrt{-\frac{q}{4 r}} \tanh a(z-\nu t)+\sqrt{\frac{q}{4 r}} \operatorname{sech} a(z-\nu t), \\
& a=\sqrt{\frac{q}{2 p}}, \nu=-\frac{3 \sqrt{p q}}{\sqrt{2}} \\
& u_{4}(z, t)=-\sqrt{-\frac{q}{4 r}}-\sqrt{-\frac{q}{4 r}} \tanh a(z-\nu t)+\sqrt{\frac{q}{4 r}} \operatorname{sech} a(z-\nu t), \\
& a=\sqrt{\frac{q}{2 p}}, \nu=\frac{3 \sqrt{p q}}{\sqrt{2}} \\
& u_{5}(z, t)=\sqrt{-\frac{q}{4 r}}+\sqrt{-\frac{q}{4 r}} \tanh a(z-\nu t)+\sqrt{\frac{q}{4 r}} \operatorname{sech} a(z-\nu t), \\
& a=-\sqrt{\frac{q}{2 p}}, \nu=-\frac{3 \sqrt{p q}}{\sqrt{2}} \\
& u_{6}(z, t)=-\sqrt{-\frac{q}{4 r}}+\sqrt{-\frac{q}{4 r}} \tanh a(z-\nu t)+\sqrt{\frac{q}{4 r}} \operatorname{sech} a(z-\nu t), \\
& a=-\sqrt{\frac{q}{2 p}}, \nu=\frac{3 \sqrt{p q}}{\sqrt{2}} \\
& u_{7}(z, t)=\sqrt{-\frac{q}{4 r}}-\sqrt{-\frac{q}{4 r}} \tanh a(z-\nu t)+\sqrt{\frac{q}{4 r}} \operatorname{sech} a(z-\nu t), \\
& a=-\sqrt{\frac{q}{2 p}}, \nu=\frac{3 \sqrt{p q}}{\sqrt{2}} \\
& u_{8}(z, t)=-\sqrt{-\frac{q}{4 r}}-\sqrt{-\frac{q}{4 r}} \tanh a(z-\nu t)+\sqrt{\frac{q}{4 r}} \operatorname{sech} a(z-\nu t), \\
& a=\sqrt{\frac{q}{2 p}}, \nu=\frac{3 \sqrt{p q}}{\sqrt{2}} \\
& u_{9}(z, t)=\sqrt{-\frac{q}{4 r}}+\sqrt{-\frac{q}{4 r}} \tanh a(z-\nu t)-\sqrt{\frac{q}{4 r}} \operatorname{sech} a(z-\nu t), \\
& a=\sqrt{\frac{q}{2 p}}, \nu=\frac{3 \sqrt{p q}}{\sqrt{2}} \\
& u_{10}(z, t)=-\sqrt{-\frac{q}{4 r}}+\sqrt{-\frac{q}{4 r}} \tanh a(z-\nu t)-\sqrt{\frac{q}{4 r}} \operatorname{sech} a(z-\nu t), \\
& a=\sqrt{\frac{q}{2 p}}, \nu=-\frac{3 \sqrt{p q}}{\sqrt{2}} \\
& u_{11}(z, t)=\sqrt{-\frac{q}{4 r}}-\sqrt{-\frac{q}{4 r}} \tanh a(z-\nu t)-\sqrt{\frac{q}{4 r}} \operatorname{sech} a(z-\nu t), \\
& a=\sqrt{\frac{q}{2 p}}, \nu=-\frac{3 \sqrt{p q}}{\sqrt{2}} \\
& u_{12}(z, t)=-\sqrt{-\frac{q}{4 r}}-\sqrt{-\frac{q}{4 r}} \tanh a(z-\nu t)-\sqrt{\frac{q}{4 r}} \operatorname{sech} a(z-\nu t), \\
& a=\sqrt{\frac{q}{2 p}}, \nu=\frac{3 \sqrt{p q}}{\sqrt{2}} \\
& u_{13}(z, t)=\sqrt{-\frac{q}{4 r}}+\sqrt{-\frac{q}{4 r}} \tanh a(z-\nu t)-\sqrt{\frac{q}{4 r}} \operatorname{sech} a(z-\nu t), \\
& a=-\sqrt{\frac{q}{2 p}}, \nu=-\frac{3 \sqrt{p q}}{\sqrt{2}} \\
& u_{14}(z, t)=-\sqrt{-\frac{q}{4 r}}+\sqrt{-\frac{q}{4 r}} \tanh a(z-\nu t)-\sqrt{\frac{q}{4 r}} \operatorname{sech} a(z-\nu t), \\
& u_{15}(z, t)=\sqrt{-\frac{q}{4 r}}-\sqrt{-\frac{q}{4 r}} \tanh a(z-\nu t)-\sqrt{\frac{q}{4 r}} \operatorname{sech} a(z-\nu t), \\
& a=-\sqrt{\frac{q}{2 p}}, \nu=\frac{3 \sqrt{p q}}{\sqrt{2}} \\
& u_{16}(z, t)=-\sqrt{-\frac{q}{4 r}}-\sqrt{-\frac{q}{4 r}} \tanh a(z-\nu t)-\sqrt{\frac{q}{4 r}} \operatorname{sech} a(z-\nu t), \\
& a=-\sqrt{\frac{q}{2 p}}, \nu=\frac{3 \sqrt{p q}}{\sqrt{2}} \\
& a=\sqrt{\frac{q}{2 p}}, \nu=\frac{3 \sqrt{p q}}{\sqrt{2}} \\
& u_{17}(z, t)=\sqrt{-\frac{q}{4 r}}+\sqrt{-\frac{q}{4 r}} \tanh a(z-\nu t) \\
& u_{18}(z, t)=-\sqrt{-\frac{q}{4 r}}+\sqrt{-\frac{q}{4 r}} \tanh a(z-\nu t) \\
& u_{19}(z, t)=\sqrt{-\frac{q}{4 r}}-\sqrt{-\frac{q}{4 r}} \tanh a(z-\nu t) \\
& u_{20}(z, t)=-\sqrt{-\frac{q}{4 r}}-\sqrt{-\frac{q}{4 r}} \tanh a(z-\nu t), \\
& u_{21}(z, t)=\sqrt{-\frac{q}{4 r}}+\sqrt{-\frac{q}{4 r}} \tanh a(z-\nu t), \\
& u_{22}(z, t)=-\sqrt{-\frac{q}{4 r}}+\sqrt{-\frac{q}{4 r}} \tanh a(z-\nu t), \\
& u_{23}(z, t)=\sqrt{-\frac{q}{4 r}}-\sqrt{-\frac{q}{4 r}} \tanh a(z-\nu t), \\
& u_{24}(z, t)=-\sqrt{-\frac{q}{4 r}}-\sqrt{-\frac{q}{4 r}} \tanh a(z-\nu t), \\
& a=\sqrt{\frac{q}{8 p}}, \nu=\frac{3 \sqrt{p q}}{\sqrt{2}} \\
& a=\sqrt{\frac{q}{8 p}}, \nu=-\frac{3 \sqrt{p q}}{\sqrt{2}} \\
& a=\sqrt{\frac{q}{8 p}}, \nu=-\frac{3 \sqrt{p q}}{\sqrt{2}} \\
& a=\sqrt{\frac{q}{8 p}}, \nu=\frac{3 \sqrt{p q}}{\sqrt{2}} \\
& a=-\sqrt{\frac{q}{8 p}}, \nu=\frac{3 \sqrt{p q}}{\sqrt{2}} \\
& a=-\sqrt{\frac{q}{8 p}}, \nu=-\frac{3 \sqrt{p q}}{\sqrt{2}} \\
& a=-\sqrt{\frac{q}{8 p}}, \nu=-\frac{3 \sqrt{p q}}{\sqrt{2}} \\
& a=-\sqrt{\frac{q}{8 p}}, \nu=\frac{3 \sqrt{p q}}{\sqrt{2}}
\end{aligned}
$$


A particular form of the solution $u_{17}(z, t)$ for $p=10, q=20$ and $r=-5$ is pictured out in 1 for some finite space and time domains. The initial wave profile with property $u(z, t)=2$ as $z \rightarrow \infty$ and $u(z, t)=0$, as $z \rightarrow-\infty$ moves to the right along the space axis with a constant speed. During propagation, it preserves its shape and height.

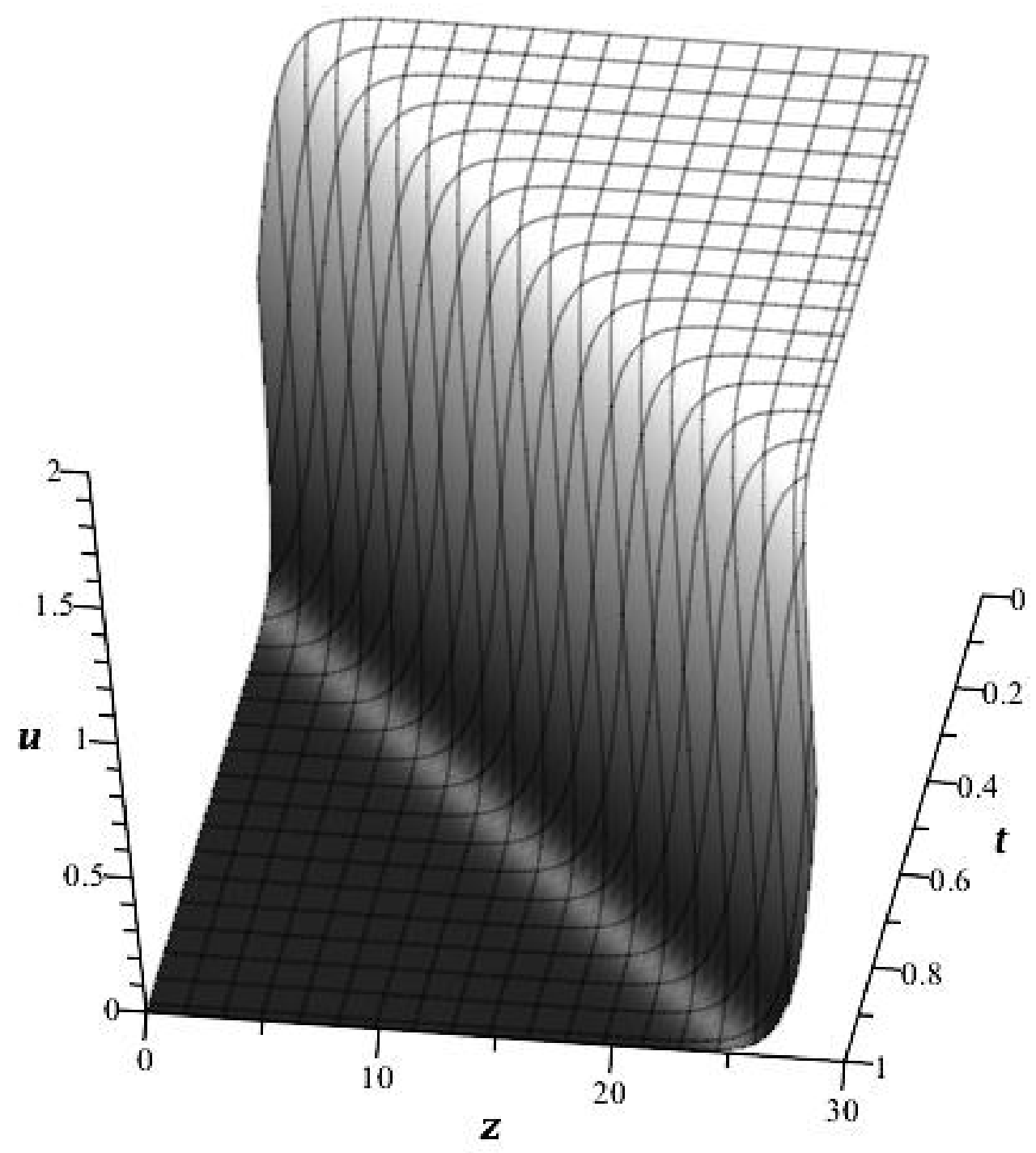

Figure 1: A particular solution of $u_{17}(z, t)=1+\frac{1}{2} \tanh \left(\frac{z}{2}-15 t\right)$ 


\section{Solutions to the $\mathrm{ZE}$}

The traveling wave transform (3) reduces the $\mathrm{ZE}(2)$ to

$$
-a \nu U^{\prime}+p a^{2} U^{\prime \prime}+q U^{2}+r U^{3}=0
$$

where ' denotes $d U / d \eta$. Since the balancing number of $U^{\prime \prime}$ and $U^{3}$ is 1 , the predicted solution should be in the form

$$
U(\eta)=A_{0}+A_{1} \tanh \eta+B_{1} \operatorname{sech} \eta
$$

or

$$
U(w)=A_{0}-A_{1} \cos w+B_{1} \sin w
$$

Substitution of this solution into (19) is the next step. Some simplifications using some trigonometric relations and equating the powers of trigonometric functions to zero give the set of algebraic equations

$$
\begin{aligned}
r A_{0}{ }^{3}+3 r A_{0} B_{1}{ }^{2}-a \nu A_{1}+q A_{0}{ }^{2}+q B_{1}{ }^{2} & =0 \\
-a^{2} p B_{1}+3 r A_{0}{ }^{2} B_{1}+r B_{1}{ }^{3}+2 q A_{0} B_{1} & =0 \\
-2 a^{2} p A_{1}-r A_{1}{ }^{3}+3 r A_{1} B_{1}{ }^{2} & =0 \\
3 r A_{0} A_{1}{ }^{2}-3 r A_{0} B_{1}{ }^{2}+a \nu A_{1}+q A_{1}{ }^{2}-q B_{1}{ }^{2} & =0 \\
2 a^{2} p B_{1}+3 r A_{1}{ }^{2} B_{1}-r B_{1}{ }^{3} & =0 \\
-6 r A_{0} A_{1} B_{1}-a \nu B_{1}-2 q A_{1} B_{1} & =0 \\
2 a^{2} p A_{1}-3 r A_{0}{ }^{2} A_{1}-3 r A_{1} B_{1}{ }^{2}-2 q A_{0} A_{1} & =0
\end{aligned}
$$


Solution of this system of equations gives

$$
\begin{aligned}
& a=\sqrt{-\frac{1}{2 p r}} q, \quad \nu=-\frac{q}{2 r \sqrt{-\frac{1}{2 p r}}}, A_{0}=-\frac{q}{2 r}, \quad A_{1}=-\frac{q}{2 r}, B_{1}=\frac{q}{2 r} i \\
& a=-\sqrt{-\frac{1}{2 p r}} q, \quad \nu=\frac{q}{2 r \sqrt{-\frac{1}{2 p r}}}, A_{0}=-\frac{q}{2 r}, \quad A_{1}=-\frac{q}{2 r}, B_{1}=\frac{q}{2 r} i \\
& a=\sqrt{-\frac{1}{2 p r}} q, \quad \nu=-\frac{q}{2 r \sqrt{-\frac{1}{2 p r}}}, A_{0}=-\frac{q}{2 r}, \quad A_{1}=-\frac{q}{2 r}, B_{1}=-\frac{q}{2 r} i \\
& a=-\sqrt{-\frac{1}{2 p r}} q, \quad \nu=\frac{q}{2 r \sqrt{-\frac{1}{2 p r}}}, A_{0}=-\frac{q}{2 r}, \quad A_{1}=-\frac{q}{2 r}, B_{1}=-\frac{q}{2 r} i \\
& a=\sqrt{-\frac{1}{2 p r}} q, \quad \nu=-\frac{q}{2 r \sqrt{-\frac{1}{2 p r}}}, A_{0}=-\frac{q}{2 r}, \quad A_{1}=\frac{q}{2 r}, B_{1}=\frac{q}{2 r} i \\
& a=-\sqrt{-\frac{1}{2 p r}} q, \quad \nu=\frac{q}{2 r \sqrt{-\frac{1}{2 p r}}}, A_{0}=-\frac{q}{2 r}, \quad A_{1}=\frac{q}{2 r}, B_{1}=\frac{q}{2 r} i \\
& a=\sqrt{-\frac{1}{2 p r}} q, \quad \nu=-\frac{q}{2 r \sqrt{-\frac{1}{2 p r}}}, A_{0}=-\frac{q}{2 r}, \quad A_{1}=\frac{q}{2 r}, B_{1}=-\frac{q}{2 r} i \\
& a=-\sqrt{-\frac{1}{2 p r}} q, \quad \nu=\frac{q}{2 r \sqrt{-\frac{1}{2 p r}}}, A_{0}=-\frac{q}{2 r}, \quad A_{1}=\frac{q}{2 r}, B_{1}=-\frac{q}{2 r} i \\
& a=\sqrt{-\frac{1}{8 p r}} q, \quad \nu=\frac{q}{4 r \sqrt{-\frac{1}{8 p r}}}, A_{0}=-\frac{q}{2 r}, \quad A_{1}=-\frac{q}{2 r}, B_{1}=0 \\
& a=-\sqrt{-\frac{1}{8 p r}} q, \quad \nu=-\frac{q}{4 r \sqrt{-\frac{1}{8 p r}}}, A_{0}=-\frac{q}{2 r}, \quad A_{1}=-\frac{q}{2 r}, B_{1}=0 \\
& a=\sqrt{-\frac{1}{8 p r}} q, \quad \nu=\frac{q}{4 r \sqrt{-\frac{1}{8 p r}}}, A_{0}=-\frac{q}{2 r}, \quad A_{1}=-\frac{q}{2 r}, B_{1}=0 \\
& a=-\sqrt{-\frac{1}{8 p r}} q, \quad \nu=-\frac{q}{4 r \sqrt{-\frac{1}{8 p r}}}, A_{0}=-\frac{q}{2 r}, \quad A_{1}=-\frac{q}{2 r}, B_{1}=0
\end{aligned}
$$


Thus, the solutions to the ZE (2) are expressed explicitly as

$$
\begin{aligned}
& u_{25}(z, t)=-\frac{q}{2 r}-\frac{q}{2 r} \tanh (a(z-\nu t))+\frac{q}{2 r} i \operatorname{sech}(a(z-\nu t)), \quad a=\sqrt{-\frac{1}{2 p r}} q, \nu=-\frac{q}{2 r \sqrt{-\frac{1}{2 p r}}} \\
& u_{26}(z, t)=-\frac{q}{2 r}-\frac{q}{2 r} \tanh (a(z-\nu t))+\frac{q}{2 r} i \operatorname{sech}(a(z-\nu t)), \quad a=-\sqrt{-\frac{1}{2 p r}} q, \nu=\frac{q}{2 r \sqrt{-\frac{1}{2 p r}}} \\
& u_{27}(z, t)=-\frac{q}{2 r}-\frac{q}{2 r} \tanh (a(z-\nu t))-\frac{q}{2 r} i \operatorname{sech}(a(z-\nu t)), \quad a=\sqrt{-\frac{1}{2 p r}} q, \nu=-\frac{q}{2 r \sqrt{-\frac{1}{2 p r}}} \\
& u_{28}(z, t)=-\frac{q}{2 r}-\frac{q}{2 r} \tanh (a(z-\nu t))-\frac{q}{2 r} i \operatorname{sech}(a(z-\nu t)), \quad a=-\sqrt{-\frac{1}{2 p r}} q, \nu=\frac{q}{2 r \sqrt{-\frac{1}{2 p r}}} \\
& u_{29}(z, t)=-\frac{q}{2 r}+\frac{q}{2 r} \tanh (a(z-\nu t))+\frac{q}{2 r} i \operatorname{sech}(a(z-\nu t)), \quad a=\sqrt{-\frac{1}{2 p r}} q, \nu=\frac{q}{2 r \sqrt{-\frac{1}{2 p r}}} \\
& u_{30}(z, t)=-\frac{q}{2 r}+\frac{q}{2 r} \tanh (a(z-\nu t))+\frac{q}{2 r} i \operatorname{sech}(a(z-\nu t)), \quad a=-\sqrt{-\frac{1}{2 p r}} q, \nu=-\frac{q}{2 r \sqrt{-\frac{1}{2 p r}}} \\
& u_{31}(z, t)=-\frac{q}{2 r}+\frac{q}{2 r} \tanh (a(z-\nu t))-\frac{q}{2 r} i \operatorname{sech}(a(z-\nu t)), \quad a=\sqrt{-\frac{1}{2 p r}} q, \nu=\frac{q}{2 r \sqrt{-\frac{1}{2 p r}}} \\
& u_{32}(z, t)=-\frac{q}{2 r}+\frac{q}{2 r} \tanh (a(z-\nu t))-\frac{q}{2 r} i \operatorname{sech}(a(z-\nu t)), \quad a=-\sqrt{-\frac{1}{2 p r}} q, \nu=-\frac{q}{2 r \sqrt{-\frac{1}{2 p r}}} \\
& u_{33}(z, t)=-\frac{q}{2 r}-\frac{q}{2 r} \tanh (a(z-\nu t)), \\
& u_{34}(z, t)=-\frac{q}{2 r}-\frac{q}{2 r} \tanh (a(z-\nu t)), \\
& a=\sqrt{-\frac{1}{8 p r}} q, \nu=-\frac{q}{4 r \sqrt{-\frac{1}{8 p r}}} \\
& a=-\sqrt{-\frac{1}{8 p r}} q, \nu=\frac{q}{4 r \sqrt{-\frac{1}{8 p r}}} \\
& u_{35}(z, t)=-\frac{q}{2 r}+\frac{q}{2 r} \tanh (a(z-\nu t)), \\
& a=\sqrt{-\frac{1}{8 p r}} q, \nu=-\frac{q}{4 r \sqrt{-\frac{1}{8 p r}}} \\
& u_{36}(z, t)=-\frac{q}{2 r}+\frac{q}{2 r} \tanh (a(z-\nu t)), \\
& a=-\sqrt{-\frac{1}{8 p r}} q, \nu=-\frac{q}{4 r \sqrt{-\frac{1}{8 p r}}}
\end{aligned}
$$

A particular solution of $u_{36}(z, t)$ is pictured out for $q=-10, r=1$ and $p=-1$ in a finite domains of independent variables, Fig 2 . The initial wave with property $u \rightarrow 10$ as $z \rightarrow-\infty$ and $u \rightarrow 0$ as $z \rightarrow \infty$ travels with a constant speed along the space axis $z$. It preserves its height and shape during travel. 


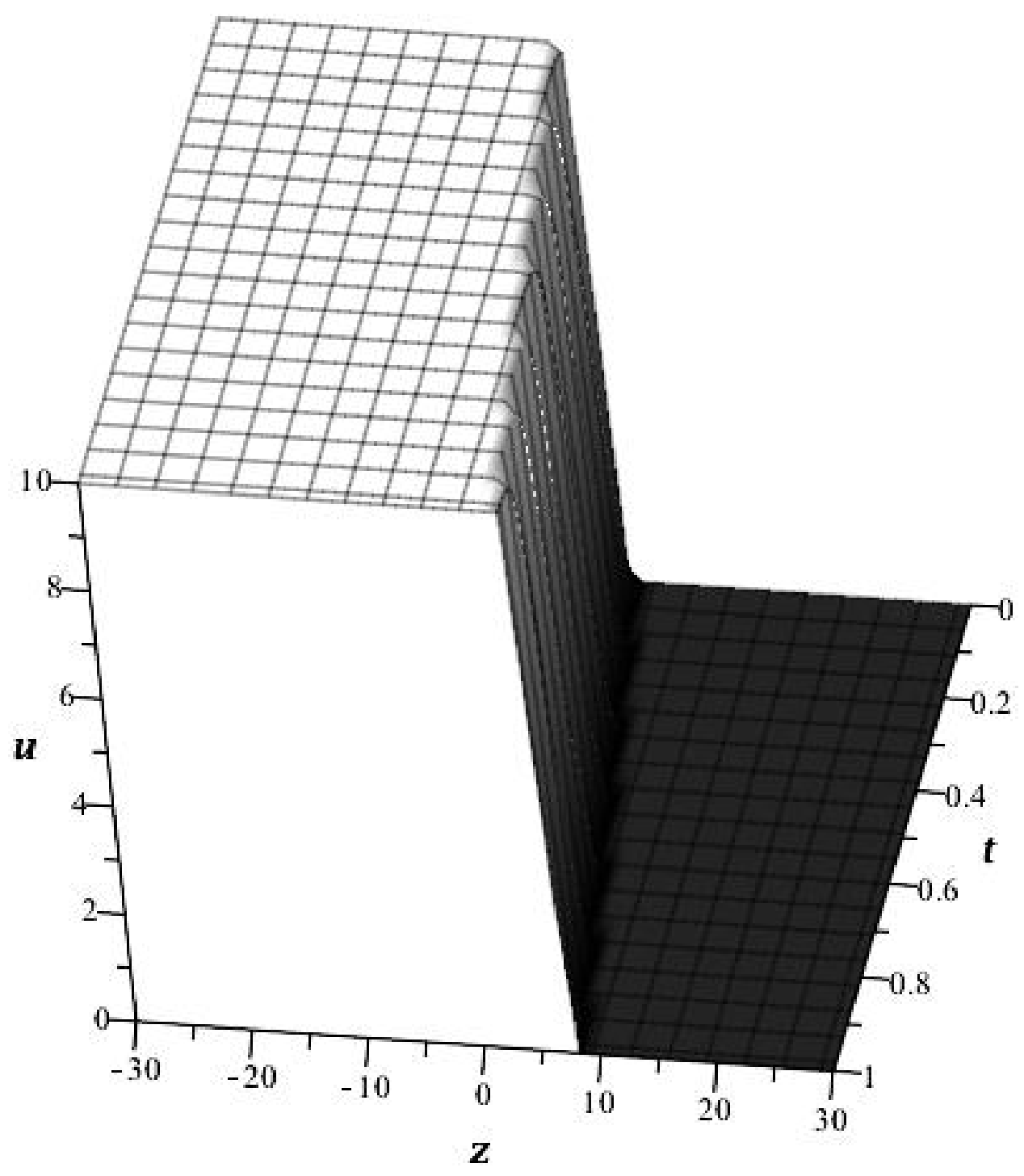

Figure 2: A particular solution of $u_{36}(z, t)=5+5 \tanh \left(-\frac{5 \sqrt{2}}{2}(z-5 \sqrt{2} t)\right)$

\section{Conclusion}

The relation between trigonometric and hyperbolic functions with help of traveling wave transform obtained from Sine-Gordon equation is used as manipulator in a finite series of hyperbolic function type solution. A plenty of real and complex valued explicit solutions to both NWSE and ZE that are significant model equa- 
tions in mathematical biology are determined by the approach. Some real valued solutions are pictured out. 


\section{References}

[1] Gilding, B.H., Kersner R. (2001), Traveling Waves in Nonlinear Diffusionconvection-reaction, University of Twente, Memorandum No. 1585.

[2] Newell, A. C., \& Whitehead, J. A. (1969). Finite bandwidth, finite amplitude convection. Journal of Fluid Mechanics, 38(2), 279-303.

[3] Segel, A. L. (1969). Distant side-walls cause slow amplitude modulation of cellular convection, J. Fluid Mech., 38(1), 203-224.

[4] Meiron, D., \& Newell, A. C. (1985). The shape of stationary dislocations. Physics Letters A, 113(5), 289-292.

[5] Nepomnyashcy, A. A., \& Pismen, L. M. (1991). Singular solutions of the nonlinear phase equation in pattern-forming systems. Physics Letters A, 153(8), 427-430.

[6] Malomed, B. A., Nepomnyashchy, A. A., \& Tribelsky, M. I. (1990). Domain boundaries in convection patterns. Physical Review A, 42(12), 7244.

[7] Malomed, B.A. (1998). Stability and Grain Boundaries in the Dispersive Newell-Whitehead-Segel Equation, Physica Scripta, 57, 115-117.

[8] Graham, R. (1996). Systematic derivation of a rotationally covariant extension of the two-dimensional Newell-Whitehead-Segel equation. Physical review letters, 76(12), 2185.

[9] Zemskov, E. P. (2014). Turing patterns and Newell-Whitehead-Segel amplitude equation. Physics-Uspekhi, 57(10), 1035.

[10] Caraballo, T., Crauel, H., Langa, J., \& Robinson, J. (2007). The effect of noise on the Chafee-Infante equation: a nonlinear case study. Proceedings of the American Mathematical Society, 135(2), 373-382.

[11] Saravanan, A., \& Magesh, N. (2013). A comparison between the reduced differential transform method and the Adomian decomposition method for the Newell-Whitehead-Segel equation. Journal of the Egyptian Mathematical Society, 21(3), 259-265.

[12] Prakash, A., \& Kumar, M. (2016). He's variational iteration method for the solution of nonlinear Newell-Whitehead-Segel equation. Journal of Applied Analysis and Computation, 6(3), 738-748. 
[13] Danilov, V.G., Maslov, V.P., Volosov, K.A., Mathematical Modelling of Heat and Mass Transfer Processes, Kluwer Academic Publishers, Dordrecht, The Netherlands, 1995.

[14] Yan, C. (1996), A simple transformation for nonlinear waves, Physics Letters A, 224, 77-84. 